(C) 2016 IEEE. Personal use of this material is permitted. Permission from IEEE must be obtained for all other uses, in any current or future media, including reprinting/republishing this material for advertising or promotional purposes, creating new collective works, for resale or redistribution to servers or lists, or reuse of any copyrighted component of this work in other works. 


\section{Range-Only Underwater Target Localization: Path Characterization}

\author{
I. Masmitja, S. Gomariz, J. Del Rio \\ SARTI Research Group. Electronics Department \\ Universitat Politècnica de Catalunya \\ Vilanova i la Geltrú, Spain \\ ivan.masmitja@upc.edu
}

\author{
B. Kieft, T. O’Reilly \\ Monterey Bay Aquarium Research Institute (MBARI) \\ Moss Landing, California, U.S.A. \\ bkieft@mbari.org
}

\begin{abstract}
Underwater localization using acoustic signals is one of the main components in a navigation system for an AUV as a more accurate alternative to dead-reckoning techniques. While different methods based on the idea of multiple beacons have been studied, other approaches use only one beacon, which reduces the system costs and deployment complexity. The inverse approach for single-beacon navigation is to use this method for target localization by an underwater or surface vehicle. In this paper we present a method of range-only target localization using a Wave Glider ${ }^{\mathrm{TM}}$, for which simulations and sea tests have been conducted to determine optimal parameters to minimize acoustic energy use and search time and to maximize location accuracy and precision.
\end{abstract}

Keywords - target localization, underwater, vehicle, acoustic

\section{INTRODUCTION}

One of the main challenges in oceanographic research is that of underwater positioning. It is well known that the GPS signal suffer a large attenuation underwater. Therefore, different methods and architectures have been developed using acoustic signals, which have better underwater performance, such as Long Baseline (LBL), Ultra Short Baseline (USBL) and GPS Intelligent Buoys (GIB). Each of these systems has its own application as a function of project necessities and constraints. For example LBL system offer the best precision and accuracy, but with high deployment and maintenances costs. These costs can be somewhat reduced by GIB systems, which use surface buoys instead of seafloor nodes. If the main goal is to reduce the setup time, the best option is a USBL system, but with less accuracy than the other methods.

The most recent works are focused on Underwater Acoustic Networks (UWAN), which implement traditional concepts of acoustic positioning. On the other hand, some studies have focused on single beacon localization methods to reduce deployment costs. In these architectures the main goal is to use one autonomous mobile beacon to localize different underwater targets. This methodology, known as single-beacon range-only localization [1][2][3][4] has its particular challenges, such as path characterization (path shape, number of points and maximum range) or performance evaluation (accuracy and reliability). All of these parameters must be evaluated under different circumstances and setup characteristics.

In the literature different papers about observability (which introduces some restrictions in paths and maneuvers) can be found, for example in [5] the authors derive that the best trajectory is to do turning motions around the beacon, and in [3] a similar approach is used with a surface vehicle following three AUVs. However, all these works are mathematical developments and only show some simulations. On the other hand, in other works such as [4] and [6] the authors present some field test results to localize an underwater target using rangeonly methods, but in their case, they do not present a general study to find the best parameters for target localization.

In this work we show how to determine the optimal parameters for this method. Additionally, we present results of simulations and sea tests to demonstrate the good performance of a Wave Glider used as a single-beacon LBL system for target localization. This method can be used in a wide range of applications using the long-duration, autonomous navigation and computational characteristics of Wave Glider applications.

A) Target localization in benthic zone:

- $\quad$ Instruments on seabed, which may be stationary or moving (e.g. slowly sliding down a submarine canyon, or on a benthic 'rover')

- $\quad$ Low motion tagged benthic marine species

B) Target localization in Pelagic zone:

- $\quad$ Drifter buoys

- $\quad$ Autonomous underwater vehicles (AUV)

- $\quad$ Low motion tagged pelagic marine species

\section{RANGE-ONLY TARGET LOCALIZATION}

The concept of single-beacon range-only positioning can be divided into two groups: as a navigational aid for a moving vehicle [7] (group 1), or to localize a stationary or moving target [1] (group 2). All these methods use a set of ranges between a target and different static nodes, known as anchor nodes. Typically, these ranges can be obtained using Time of Flight (TOF) given the speed of sound in water. Then, the unknown underwater target position problem can be solved using trilateration, where in general, three or more points are needed in 2D dimensions and, at least, four points in 3D scenarios.

In general, the inverse problem has received more attention in the literature (group 1) where an AUV needs to be located using a set of known transponders, as in [2]. However similar approaches can be used in this case, where an autonomous vehicle is used to find an underwater target (group 2). The 
method used in this paper can be seen in Fig. 1, where a rangeonly target localization method based on single-beacon architecture is presented. The target position is computed using a Wave Glider, which periodically measures the range to the underwater target, while it is moving on the surface.

Therefore, following the same notation as [2], the underwater target positioning vector can be defined as $\boldsymbol{p}_{T} \in$ $\mathbb{R}^{\boldsymbol{n}}$, where $n$ can be either 2 or 3 and is the space dimension of the problem. All the Wave Glider positions used in the trilateration problem can be denoted as $\boldsymbol{p}_{i} \in \mathbb{R}^{\boldsymbol{n}}$ where $i \in$ $\{1,2, \ldots, m\}$. Then, the ranges measured with Wave Glider between itself and target can be expressed as

$$
\bar{r}_{i}=\left\|\boldsymbol{p}_{T}-\boldsymbol{p}_{i}\right\|+w_{i}, \quad i \in\{1,2, \ldots, m\}
$$

where $\left\|\boldsymbol{p}_{T}-\boldsymbol{p}_{i}\right\|=r_{i}$ is the true range and $w_{i} \sim \mathcal{N}\left(0, \sigma^{2}\right)$ is some zero mean Gaussian measurement error where $\sigma^{2}$ is the variance. Also, this expression can be written in matrix form as $\overline{\boldsymbol{r}}=\boldsymbol{r}+\boldsymbol{w}$. In general, this non-linear, non-smooth and overdetermined (when $m>n+1$ ) system doesn't have a straightforward solution. At this point, two different methodologies are used in the literature to solve the system and find the target position through ranges: linearize the function and find a closed-form Least Squares (LS) solution; or use an iterative minimization algorithm to minimize a cost function related to the Maximum Likelihood (ML) estimate.

\section{A. Closed-form Least Squares algorithm}

Because the main goal of this paper is not to compare different algorithms performance, a simple Unconstrained Least Square algorithm is used, which was introduced in [8]. However, as will be shown, its performance is quite good.

The main idea on LS algorithms is linearize the system using the squared range measurements, obtaining

$$
\bar{d}=\boldsymbol{d}+\boldsymbol{\xi}
$$

where $\boldsymbol{\xi}$ is the new measurement error as a function of $\boldsymbol{w}$ and $\boldsymbol{r}$. In this case, is not obvious that $\xi \sim \mathcal{N}\left(0, \boldsymbol{\sigma}^{2}\right)$ as before. Under some circumstances this assumption is possible, for example when $r_{i} \gg \sigma_{i}$, but this assumption is not true when the vehicle is close to the target. See [2] for more information.

On the other hand, a 2D formulation can be done in this case, where all the points used to compute the underwater target

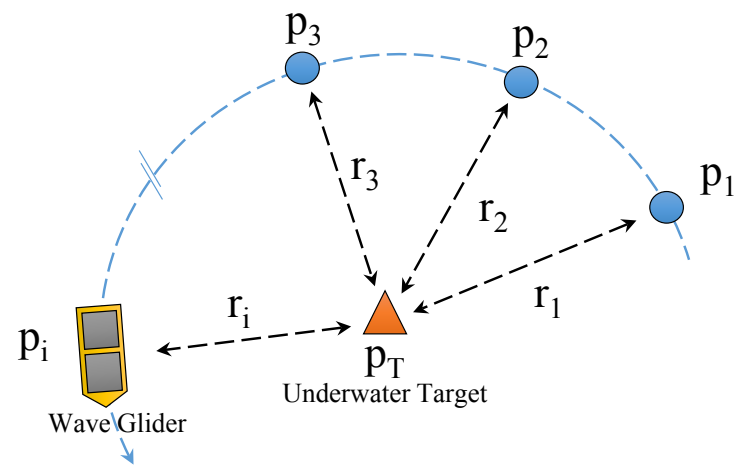

Fig. 1. Range-only single-beacon underwater target localization methodology representation, using a Wave Glider as a moving $L B L$ position are coplanar, in the same z-coordinate, which in this case is on the sea surface. The square ranges are defined by

$$
\begin{aligned}
d_{i} & =\left\|\boldsymbol{P}_{T}-\boldsymbol{P}_{\boldsymbol{i}}\right\|^{2} \\
& =\left(p_{T x}-p_{i x}\right)^{2}+\left(p_{T y}-p_{i y}\right)^{2}+\left(p_{T z}-p_{i z}\right)^{2} \\
& =\left\|\boldsymbol{p}_{T}^{\prime}-\boldsymbol{p}_{i}^{\prime}\right\|^{2}+\delta_{z}^{2} \\
& =\boldsymbol{p}_{\boldsymbol{i}}^{\prime T} \boldsymbol{p}_{\boldsymbol{i}}^{\prime}-2 \boldsymbol{p}_{\boldsymbol{i}}^{\prime T} \boldsymbol{p}_{T}^{\prime}+\left\|\boldsymbol{p}_{T}^{\prime}\right\|^{2}+\delta_{z}^{2}
\end{aligned}
$$

and

$$
\boldsymbol{d}=\delta\left(\boldsymbol{P}^{\prime T} \boldsymbol{P}^{\prime}\right)-2 P^{\prime T} \boldsymbol{p}_{T}^{\prime}+\left(\left\|\boldsymbol{p}_{T}^{\prime}\right\|^{2}+\delta_{z}^{2}\right) 1_{m}
$$

The unknown scalar terms $\left\|\boldsymbol{p}_{T}^{\prime}\right\|^{2}+\delta_{z}^{2}$ are multiplying the vector of ones $1_{m}$. Therefore, this unknown term can be deleted multiplying both sides of the equation by matrix $\boldsymbol{M}$, which has $1_{m}$ in his null space, obtaining

$$
\boldsymbol{M d}=\boldsymbol{M} \delta\left(\boldsymbol{P}^{\prime T} \boldsymbol{P}^{\prime}\right)-2 \boldsymbol{M} P^{\prime T} \boldsymbol{p}_{T}^{\prime}=\boldsymbol{M} \boldsymbol{d}^{\prime}
$$

Consequently, the square range in $2 \mathrm{D}$ is the same as in $3 \mathrm{D}$ and the same algorithm can be used. In this situation the depth of the target is not necessary to obtain its $(\mathrm{x}, \mathrm{y})$ position. Therefore, the depth can be computed using Pythagoras' theorem.

Finally, (4) can be written as a linear system with form $\boldsymbol{A} \boldsymbol{\theta}=\boldsymbol{b}+\boldsymbol{\xi}$, which can be solved by minimizing as small as possible the length of the error, with solution $\boldsymbol{A}^{T} \boldsymbol{A} \widehat{\boldsymbol{\theta}}=\boldsymbol{A}^{T} \boldsymbol{b}$ . Therefore, the target position estimation is

$$
\widehat{\boldsymbol{p}}_{T}=\boldsymbol{N}\left(\boldsymbol{A}^{T} \boldsymbol{A}\right)^{-1} \boldsymbol{A}^{T} \boldsymbol{b}
$$

where

$$
\begin{aligned}
\boldsymbol{N} & =\left[\begin{array}{ll}
\boldsymbol{I}_{n} & 0
\end{array}\right] \\
\boldsymbol{A} & =\left[\begin{array}{cc}
2 \boldsymbol{p}_{1}^{T} & -1 \\
\vdots & \vdots \\
2 \boldsymbol{p}_{m}^{T} & -1
\end{array}\right] \\
b & =\left[\begin{array}{c}
\left\|\boldsymbol{p}_{1}\right\|^{2}-\bar{d}_{1} \\
\vdots \\
\left\|\boldsymbol{p}_{m}\right\|^{2}-\bar{d}_{m}
\end{array}\right] \\
\theta & =\left[\begin{array}{c}
\boldsymbol{p}_{T} \\
\left\|\boldsymbol{p}_{T}\right\|^{2}
\end{array}\right]
\end{aligned}
$$

\section{B. Iterative minimization algorithm}

The main goal of this method is to use the Maximum Likelihood Estimation (MLE), a statistical technique to compute the value that maximizes the similarity between selected values and observed data, which come with an unknown probability density function. For a normal distribution and using the loglikelihood function, which is a continuous strictly increasing function over the range of the likelihood, the log-likelihood can be written as

$$
\begin{aligned}
\log \mathcal{L}\left(\boldsymbol{p}_{T}\right) & =-\frac{m}{2} \log 2 \pi-\frac{1}{2} \log |\boldsymbol{R}|-\frac{1}{2}(\overline{\boldsymbol{r}}-\boldsymbol{r})^{T} R^{-1}(\overline{\boldsymbol{r}}-\boldsymbol{r}) \\
& =K-\frac{1}{2}(\overline{\boldsymbol{r}}-\boldsymbol{r})^{T} \boldsymbol{R}^{-1}(\overline{\boldsymbol{r}}-\boldsymbol{r})
\end{aligned}
$$

where $\boldsymbol{R}$ is a diagonal matrix, which values are the measurement error covariance $\sigma^{2}$. Then the MLE can be found by solving the 
optimization problem $\hat{\theta}=\arg \min _{\boldsymbol{p}_{T}} f\left(\boldsymbol{p}_{T}\right)$, where the cost function is

$$
f\left(\boldsymbol{p}_{T}\right):=\frac{1}{2}(\overline{\boldsymbol{r}}-\boldsymbol{r})^{T} \boldsymbol{R}^{-1}(\overline{\boldsymbol{r}}-\boldsymbol{r})
$$

In general, this cost function is non-linear because of the square root that defines the range measurements, therefore there is no closed form solution. However, an iterative method can be used to solve this minimization problem, such as negative gradient descent or Newton methods. Only the final formulation is presented in this paper to reduce its length, for detailed development see [2] and [9].

To use these two iterative minimization methods the cost function gradient and its Hessian must be calculated, obtaining

$$
\nabla f\left(\boldsymbol{p}_{T}\right)=-\boldsymbol{C} \delta(\boldsymbol{r})^{-1} \boldsymbol{R}^{-1}(\overline{\boldsymbol{r}}-\boldsymbol{r})
$$

And

$\nabla^{2} f\left(\boldsymbol{p}_{T}\right)$

$$
=-\boldsymbol{C} \delta(\boldsymbol{r})^{-3} \boldsymbol{R}^{-1} \delta(2 \boldsymbol{r}-\overline{\boldsymbol{r}}) \boldsymbol{C}^{T}+\boldsymbol{\alpha}^{T} \delta(\boldsymbol{r})^{-1} \mathbf{1}_{m} \boldsymbol{I}_{n}
$$

where

$$
\begin{gathered}
\boldsymbol{R}=\left[\begin{array}{ccc}
\sigma^{2} & \cdots & 0 \\
\vdots & \ddots & \vdots \\
0 & \cdots & \sigma^{2}
\end{array}\right] \\
\boldsymbol{\alpha}=\boldsymbol{R}^{-1}(\overline{\boldsymbol{r}}-\boldsymbol{r}) \\
\boldsymbol{C}=\left[\begin{array}{ccc}
\boldsymbol{p}_{T}-\boldsymbol{p}_{1} & \ldots & \boldsymbol{p}_{T}-\boldsymbol{p}_{m}
\end{array}\right]
\end{gathered}
$$

Using the gradient of the cost function and its Hessian the iterative minimization algorithm can be computed by Algorithm 1.

Algorithm 1: Iterative Minimization

1: Start from an initial estimation value $\boldsymbol{p}_{T 0}$. And set $k=0$

2: Calculate a search direction using Gradient descent (13) or Newton descent (14).

a) $h\left(\boldsymbol{p}_{T}\right)=-\nabla f\left(\boldsymbol{p}_{T}\right)$

b) $h\left(\boldsymbol{p}_{T}\right)=-\left(\nabla^{2} f\left(\boldsymbol{p}_{T}\right)\right)^{-1} \nabla f\left(\boldsymbol{p}_{T}\right)$

3: Determine the step size (Armijo rule).

$$
s_{k}=s \beta^{m_{i}}
$$

where $s>0, \beta, \sigma \in(0,1)$, and $m_{i}$ is the first integer that satisfies

$$
\begin{aligned}
& f\left(\boldsymbol{p}_{T k}+s \beta^{m_{i}} \mathrm{~h}\left(\boldsymbol{p}_{T k}\right)\right) \\
& \quad \leq f\left(\boldsymbol{p}_{T k}\right)+\sigma s \beta^{m_{i}} \mathrm{~h}\left(\boldsymbol{p}_{T k}\right)^{T} \nabla f\left(\boldsymbol{p}_{T k}\right)
\end{aligned}
$$

4: Update the estimation value.

$$
\begin{aligned}
\boldsymbol{p}_{T k+1} & =\boldsymbol{p}_{T k}+s_{k} h\left(\boldsymbol{p}_{T}\right) \\
k & =k+1
\end{aligned}
$$

5: if $\left\|\nabla f\left(\boldsymbol{p}_{T}\right)\right\| \leq \epsilon$ or $k \geq k_{\max }$ : stop

else: go to 2

After these mathematical formulations a set of different simulations and real tests can be conducted to characterize the performance of the system and identify the best parameters for underwater target localization using a Wave Glider with singlerange and single-beacon architecture.

\section{SimULATIONS}

Different simulations were conducted to determine the best parameters to increase the capabilities of the acoustic positioning system. Four parameters were selected: path shape, number of points needed, radius around target and offset from target. Moreover, the performance of the derived LS and MLE algorithms was compared against the Cramér-Rao Bound (CRB), which specifies the best possible performance attainable with any estimator.

For a scalar unbiased case, the variance of estimator $\widehat{\boldsymbol{p}_{T}}$ is bounded by the Fisher information $I\left(\widehat{\boldsymbol{p}_{T}}\right)$ as

$$
\operatorname{var}\left(\widehat{\boldsymbol{p}_{T}}\right) \geq 1 / I\left(\widehat{\boldsymbol{p}_{T}}\right)
$$

where the Fisher information is defined by

$$
I\left(\widehat{\boldsymbol{p}_{T}}\right)=-E\left[\partial^{2} \ell\left(r ; \boldsymbol{p}_{T}\right) / \partial \boldsymbol{p}_{T}{ }^{2}\right]
$$

where $\ell\left(r ; \boldsymbol{p}_{T}\right)=\log \mathcal{L}\left(\boldsymbol{p}_{T}\right)$, which can be seen in (11), and $E$ denotes the expected value. Computing the second derivative of the likelihood logarithm function and its expected value, the CRB obtained is

$$
\operatorname{var}\left(\widehat{\boldsymbol{p}_{T}}\right) \geq \operatorname{tr}\left[\left(\boldsymbol{C} \delta(\boldsymbol{r})^{-1} \boldsymbol{R}^{-1} \delta(\boldsymbol{r})^{-1} \boldsymbol{C}^{T}\right)^{-1}\right]
$$

which can be compared with the Root Mean Square Error (RMSE), which represents the sample standard deviation of the differences between predicted values and observed values, using the expression $R M S E=\sqrt{\operatorname{var}\left(\widehat{\boldsymbol{p}_{T}}\right)}$.

Different scenarios can be computed using (20) to observe the theoretical performance of the configuration (Wave Glider as a LBL system and an underwater target at $1800 \mathrm{~m}$ of depth). For example, Fig. 2 shows the CRB comparison between different shapes, number of points and radius from target (top, center and bottom images respectively). These figures show that the better accuracy and precision are obtained with a circle shape, long radius and large quantity of points. However, more scenarios have been simulated to obtain a better characterization. All the simulations conducted for this paper have been obtained through 1000 Monte Carlo iterations, with a normal noise probability distribution, with zero mean and standard deviation equal to 1 . 

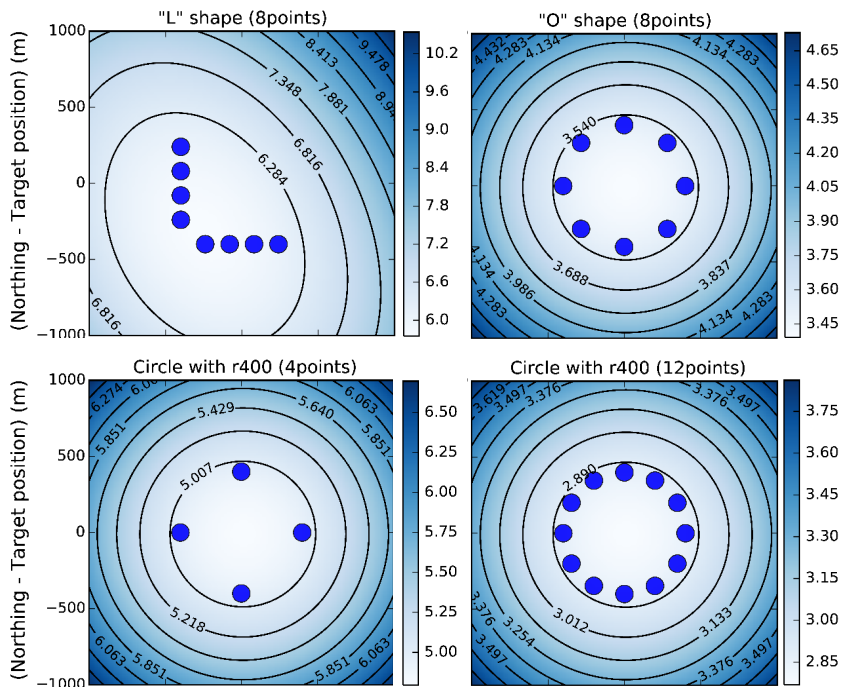

Circle with r400 (12points)
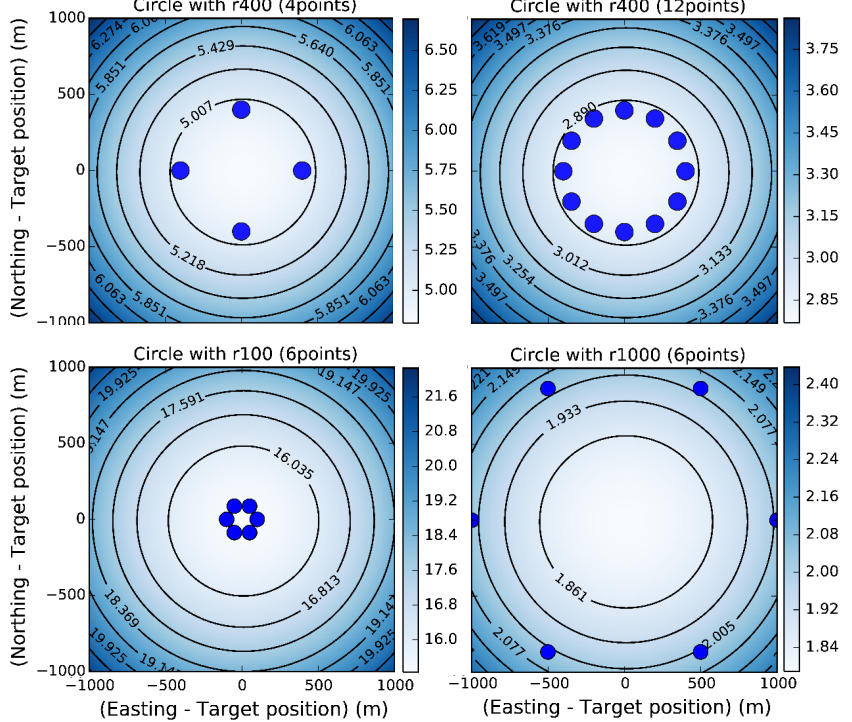

Fig. 2. Cramér-Rao Bound representation under different scenarios: comparison of different path shapes (top figures), comparison of different number of points (middle figures), and comparison of different radius (bottom figures). All these representations are for a target depth equal to $1800 \mathrm{~m}$. Blue dots represents the points used.

\section{A. Number of points}

One of the main ways to increase the precision of the system is using more points to compute the target position. This is the most common method to reduce the variance of any measurement with random noise, and is given by

$$
\operatorname{var}\left(z_{i}\right)=\frac{1}{n-1} \sum_{j=1}^{n}\left(z_{j}-\bar{z}\right)^{2}
$$

where $z_{i}$ are $n$ independent observations of $z$. This behavior can be seen in Fig. 3, which show the results for 4, 6, 12, 24 points for different radius and distances from target.

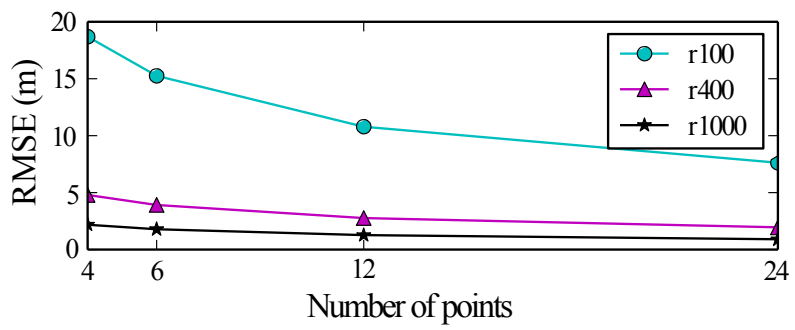

Fig. 3. RMSE evolution as a function of the number of points used to compute the target position, for circles centered over the target with
$100 \mathrm{~m}, 400 \mathrm{~m}$, and $1000 \mathrm{~m}$ of radius (r100, r400, and r1000 respectively). With $1800 \mathrm{~m}$ target depth.

\section{B. Radius around target}

Another interesting test is to observe the behavior under different path radii centered over the target, this parameter is shown in Fig. 4 where 50, 100, 200, 400, 600, 800 and 1000 meters path radius are simulated. Moreover, LS and MLE algorithms are compared against CRB. It can be observed that the performance of both algorithms are very similar and very close to the CRB. In all cases a greater radius results in a lower RMSE.

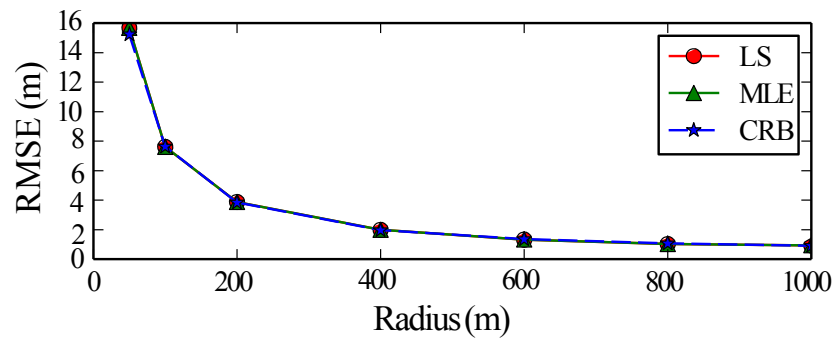

Fig. 4. RMSE evolution as a function of the circle radius, for a target at $1800 \mathrm{~m}$ of depth. Moreover, a comparison between LS, MLE and CRB algorithms are shown.

This behavior can be derived computing the surface range, which is

$$
s r=\sqrt{r^{2}-\operatorname{depth}^{2}}=\sqrt{r^{2}-(r-x)^{2}}
$$

where $x$ is the difference between range and depth. The error can be defined as $\varepsilon=(s r-\overline{s r})$ and is

$$
\varepsilon=\sqrt{2 r x-x^{2}}-\sqrt{2 r(x+w)-x^{2}+w^{2}}
$$

With (23) we can observe that if $x \simeq 0$ (depth and range are very similar) the error is $\varepsilon \simeq \sqrt{2 r w+w^{2}}$ and if $x \simeq r$ (range is much bigger than depth) the error is $\varepsilon \simeq w$.

\section{Offset from target}

Finally, a set of simulations have been conducted to observe the RMSE with different offsets between the center of the circle path and the underwater target. In this case, as before, both algorithms have the same performance as the CRB. RMSE increases with the offset between the path and target, as shown in Fig. 5.

\section{REAL FIELD TESTS}

Several sea tests have been conducted to compare and validate the algorithm's ability to locate a target, and to validate the optimal path, radius and number of points suggested by simulations. These tests have been conducted with two different seabed targets in Monterey Bay, California; a shallow water target (80 m depth) and a mid water target (1800 m depth). These target locations can be seen in Fig. 6. 


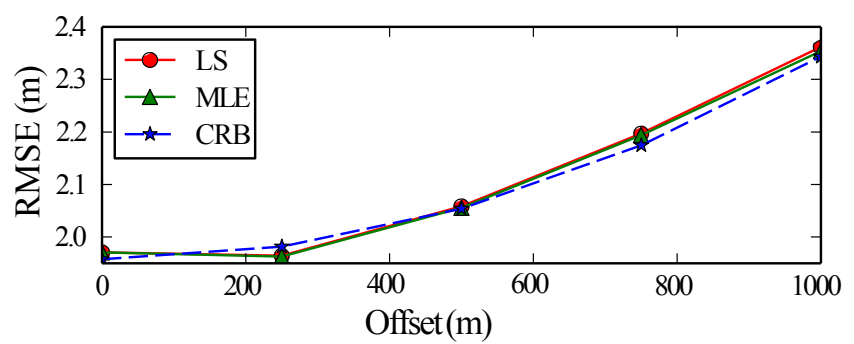

Fig. 5 RMSE evolution as a function of the offset between circumference center and target, for a target at $1800 \mathrm{~m}$ of depth. Moreover, a comparison between LS, MLE and CRB algorithms are shown.

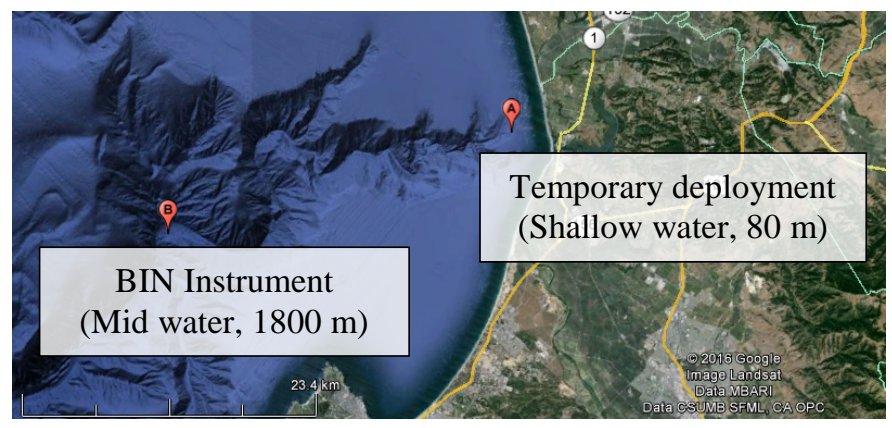

Fig. 6. Map of Monterey Bay, where the tests were carried out. These were done in two locations: first location (A) in a temporary deployment at $80 \mathrm{~m}$ depth, and a second location (B) over a BIN instrument node at $1800 \mathrm{~m}$ depth in the Monterey canyon.

In the first case (A), a set of tests was carried out to evaluate the algorithms, and the scripts to control the Wave Glider navigation. These tests were done over a temporary modem deployment in shallow water, near the coast zone at $80 \mathrm{~m}$ of depth. The path shapes realized are shown in Fig. 7; in this case a square and a circle path were used.

After these initial tests, two groups of tests were conducted over the BIN instrument node (which is deployed at $1800 \mathrm{~m}$ of depth) to determine the best radius and offset of a circle path shape. Fig. 8 shows the path shapes of the first group of tests, which consists of three circles of $100 \mathrm{~m}, 400 \mathrm{~m}$ and $800 \mathrm{~m}$ of radius, all of them centered over the BIN. Moreover the main results are shown in Table I: the target position computed using LS algorithm (easting, northing and depth), the error versus target true position, number of points used (Np), and total time to complete the path. Target true position was obtained using the average value of three paths shapes with a total of 154 ranges.

TABLE I. MAIN RESULTS FOR FIELD TEST 1

\begin{tabular}{c|ccccccccc}
\multicolumn{2}{c}{ Path } & Easting & error & Northing & error & Depth & error & Np & Time \\
\hline r100 & 580922.120 & 15.1 & 4062178.835 & -1.2 & 1860.7 & 0.7 & 11 & $13^{\prime}$ \\
$r 400$ & 580936.959 & 0.2 & 4062175.635 & 2.0 & 1858.7 & 2.7 & 36 & $56^{\prime}$ \\
$r 800$ & 580936.095 & 1.1 & 4062179.406 & -1.8 & 1863.8 & -2.4 & 64 & $1 \mathrm{~h} 57^{\prime}$
\end{tabular}

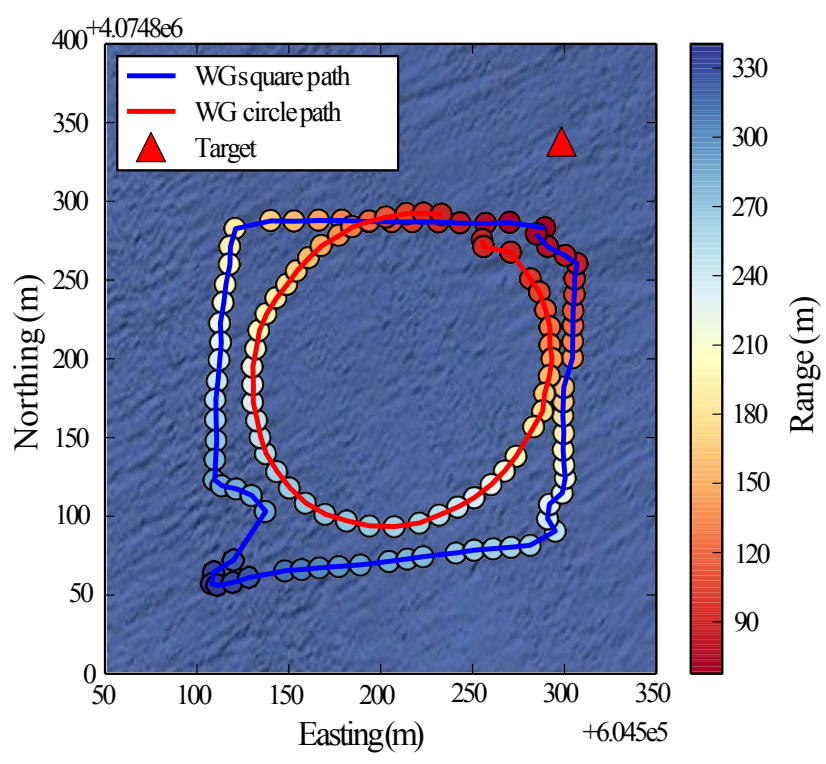

Fig. 7. Square and circle path shape, blue and red lines consecutively, done over a temporary deployment target at $80 \mathrm{~m}$ depth. Each dot represents a WG range acquired, where its color is the range value in meters. Triangle is the computed target position.

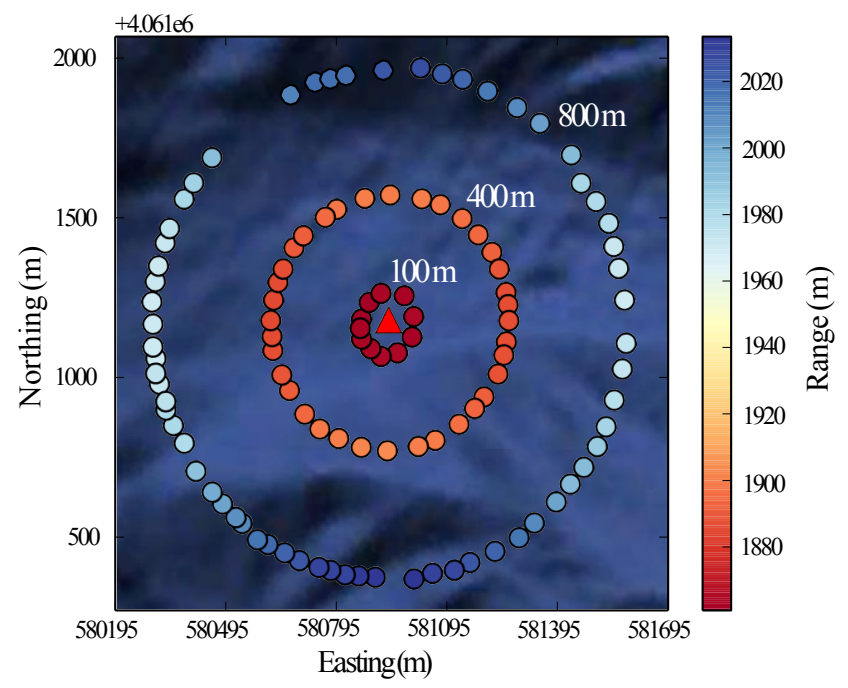

Fig. 8. WG trajectories realized over BIN target with three different radius, which were 100, 400 and 800 meters.

To compare field test results with the simulation results we compute the target position using only 4 or 6 equidistant points between all the ranges obtained in the field test, which allow us to choose different groups of 4 and 6 points and take the average value of the RMSE. These results are shown in Fig. 9. 


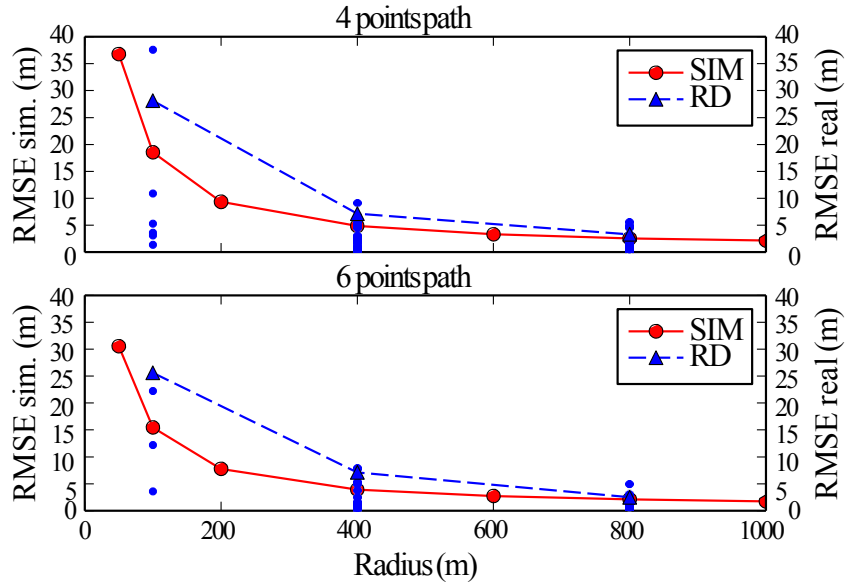

Fig. 9. These graphics show a behavior comparison between simulation (SIM) and real data (RD) results for different radius of circle paths centered over the BIN target. Using 4 and 6 equidistant points to compute the target localization.

In these graphs we can observe that the real data performance is similar to the results obtained with simulations. And with a radius equal or greater than $400 \mathrm{~m}$ we obtain good performance, with a RMSE lower than $10 \mathrm{~m}$. We can obtain a RMSE lower than $5 \mathrm{~m}$ for radius greater than $800 \mathrm{~m}$.

Finally a second field test was conducted over the BIN target to observe the accuracy influence with offset. For this purpose three paths was done, with distances of $0 \mathrm{~m}, 500 \mathrm{~m}$ and $100 \mathrm{~m}$ between circumference center and target, and with a radius of $400 \mathrm{~m}$. Fig. 10 shows the path shapes of this second test. Moreover the main results are shown in Table II.

TABLE II. MAIN RESULTS FOR FIELD TEST 2

\begin{tabular}{c|cccccccc}
\multicolumn{1}{c}{ Path } & Easting & error & Northing & error & Depth & error & Np & Time \\
\hline$d 0$ & 580936.959 & 0.2 & 4062175.635 & 2.0 & 1858.7 & 2.7 & 36 & $56^{\prime}$ \\
$d 500$ & 580946.282 & -9.1 & 4062187.115 & -9.5 & 1860.3 & 1.1 & 34 & $59^{\prime}$ \\
$d 1000$ & 580956.817 & -19.6 & 4062190.221 & -12.6 & 1856.3 & 5.1 & 43 & $1 \mathrm{~h} 4$
\end{tabular}

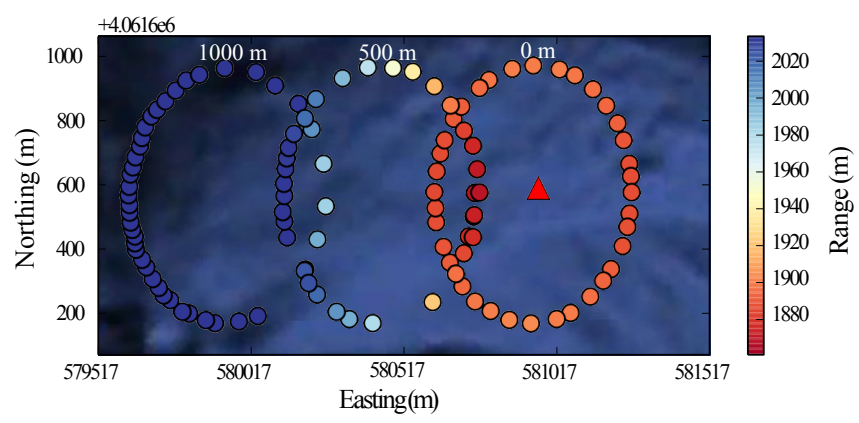

Fig. 10. WG trajectories realized over BIN target with three different offsets between target and circle center, which were 0, 500 and 1000 meters.
As before, we compute the RMSE using different groups of 4 or 6 equidistant ranges to compare the field results with simulations. These results can be observed in Fig. 11. We can see that the behavior between real tests and simulations is similar, but the RMSE obtained during the tests is bigger than the results obtained with simulations. This shows that the mathematical model for standard deviation used for simulations is not accurate, when we want to compute the target localization error, when the center of the points used as anchors are far away from the target. Therefore, a future and more detailed study for this case is necessary.

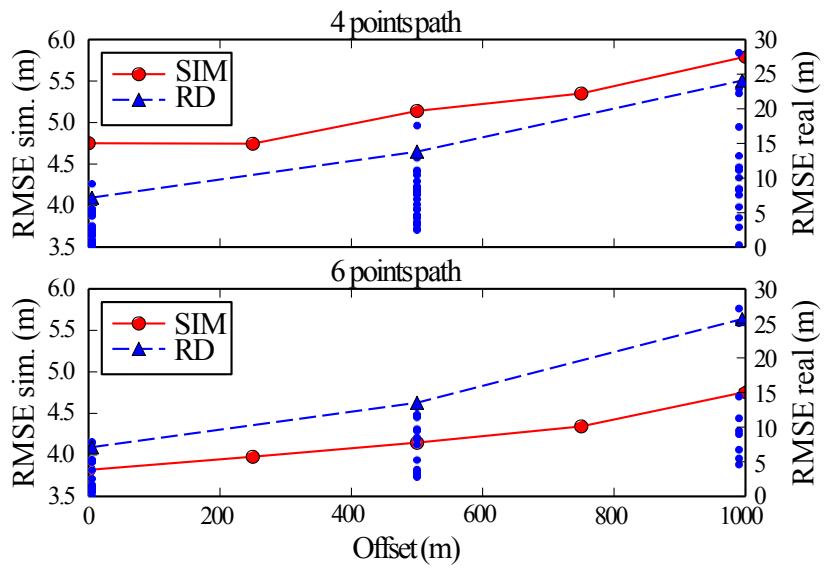

Fig. 11. These graphics show a behavior comparison between simulations (SIM) and real data (RD) results for different offsets of circle paths cover the BIN target. Using 4 and 6 equidistant points to compute the target localization.

\section{CONCLUSIONS}

This work shows the Wave Glider performance as a moving LBL with simulations and real sea tests. With this study we can determine the best path and its characteristics, such as number of points, radius or offset, to obtain the desired target localization performance, which are a minimum number of points equal to 12, radius greater than $400 \mathrm{~m}$ and offset as low as possible. With these parameters we can obtain a RMSE less than 4 meters.

Mathematical algorithms and performance have been compared with sea test, showing a good similarity, which corroborates the simulations done in this paper. However, the RMSE observed with different offsets was greater than the RMSE obtained by simulations; this might be caused by the noise model used, which was simple Gaussian noise with zero mean and standard deviation equal to one. Therefore, future improvements of this model will be studied to adjust it with the real performance observed.

Finally, future comparisons with these results with other aspects such as weather conditions or mission parameters (for example, time to complete the path or power consumption) can be done. 


\section{ACKNOWLEDGMENT}

This work was partially supported by the project NeXOS from the European Union's Seventh Programme for research, technological development and demonstration under grant agreement No 614102. We also had financial support from Spanish Ministerio de Economia y Competitividad under contract CGL2013- 42557-R (Interoperabilidad e instrumentacion de plataformas autonomas marinas para la monitorizacion sismica, INTMARSIS). The main author of this work have a grant (FPIUPC) from UPC for his $\mathrm{PhD}$ research. We gratefully acknowledge the support of MBARI and the David and Lucile Packard foundation.

\section{REFERENCES}

[1] G. Vallicrosa, P. Ridao, D. Ribas and A. Palomer, "Active Range-Only beacon localization for AUV homing," in IEEE/RSJ International Conference on Intelligent Robots and Systems, Chicago, 2014.

[2] N. nom. "Positioning and Navigation Systems for Robotic Underwater Vehicles," PhD thesis, Universidade Tecnica de Lisboa Instituto Superior Tecnico, 2009.
[3] J. D. Quenzer and K. A. Morgansen, "Observability based control in range-only underwater vehicle localization," 2014 American Control Conference, Portland, OR, 2014, pp. 4702-4707. doi: 10.1109/ACC.2014.6859032.

[4] E. Olson, J. J. Leonard and S. Teller, "Robust Range-Only Beacon Localization," in IEEE Journal of Oceanic Engineering, vol. 31, no. 4, pp. 949-958, Oct. 2006. doi: 10.1109/JOE.2006.880386.

[5] B. T. Hinson, M. K. Binder and K. A. Morgansen, "Path planning to optimize observability in a planar uniform flow field," in American Control Conference, Washington, 2013.

[6] S. D. McPhail and M. Pebody, "Range-Only Positioning of a DeepDiving Autonomous Underwater Vehicle From a Surface Ship," in IEEE Journal of Oceanic Engineering, vol. 34, no. 4, pp. 669-677, Oct. 2009. doi: 10.1109/JOE.2009.2030223.

[7] Y. T. Tan, R. Gao and M. Chitre, "Cooperative Path Planning for RangeOnly Localization Using a Single Moving Beacon," in IEEE Journal of Oceanic Engineering, vol. 39, no. 2, pp. 371-385, April 2014.

[8] K.W. Cheung, W.K Ma, and Y.T. Chan. "Least squares algorithms for time-of-arrival based mobile location," IEEE Transactions on Acoustics, Speech, and Signal Processing, 52:1121-1128, 2004.

[9] D.P. Bertsekas, "Nonlinear Programming," Athena Scientific, Belmont, MA, 1995. $2^{\text {nd }}$ edition 1999 . 